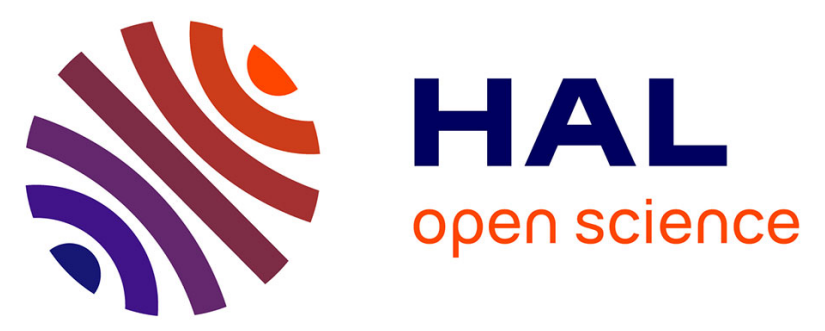

\title{
Influence du maintien en mer ou de la période du transfert en eau douce des reproducteurs de saumon Atlantique (Salmo salar) sur la maturation sexuelle et la qualité des gamètes
}

\author{
Alexis Fostier, Y. Normant, André Fauré, Maurice Loir, Bernard Jalabert, \\ Gérard Maisse, Florence Le Gac, Pierrick Haffray
}

\section{To cite this version:}

Alexis Fostier, Y. Normant, André Fauré, Maurice Loir, Bernard Jalabert, et al.. Influence du maintien en mer ou de la période du transfert en eau douce des reproducteurs de saumon Atlantique (Salmo salar) sur la maturation sexuelle et la qualité des gamètes. Aquatic Living Resources, 1995, 8, pp.135145. 10.1051/alr:1995010 . hal-02715308

\section{HAL Id: hal-02715308 \\ https: / hal.inrae.fr/hal-02715308}

Submitted on 1 Jun 2020

HAL is a multi-disciplinary open access archive for the deposit and dissemination of scientific research documents, whether they are published or not. The documents may come from teaching and research institutions in France or abroad, or from public or private research centers.
L'archive ouverte pluridisciplinaire HAL, est destinée au dépôt et à la diffusion de documents scientifiques de niveau recherche, publiés ou non, émanant des établissements d'enseignement et de recherche français ou étrangers, des laboratoires publics ou privés. 


\title{
Influence du maintien en mer ou de la période de transfert en eau douce des reproducteurs de saumon atlantique Salmo salar sur la maturation sexuelle et la qualité des gamètes
}

\author{
Pierrick Haffray (1), Alex Fostier (1), Yvon Normant ${ }^{(2)}$, André Fauré (3), \\ Maurice Loir ${ }^{(1)}$, Bernard Jalabert ${ }^{(1)}$, Gérard Maisse ${ }^{(1)}$ et Florence Le Gac ${ }^{(1)}$ \\ (1) INRA, Laboratoire de Physiologie des Poissons, Campus de Beaulieu, 35042 Rennes Cedex, France. \\ Adresse actuelle: SYSSAF, 65, nue de St-Brieuc, 35042 Rennes Cedex, France. \\ (2) IFREMER Centre de Brest, B.P. 70, 29280 Plouzané, France. \\ (3) SEMII, B.P. 17, 29450 Sizun, France.
}

Reçu le 25 juillet 1994; accepté le 9 février 1995.

Haffray P., A. Fostier, Y. Normant, A. Fauré, M. Loir, B. Jalabert, G. Maisse, F. Le Gac. Aquat. Living Resour., 1995, 8, 135-145.

Impact of sea water rearing or freshwater transfer on final maturation and on gamete quality in Atlantic salmon Salmo salar.

Abstract

Breeding management of Atlantic salmon generally requires transfer of spawners to fresh water prior to final sexual maturation, in order to avoid poor gamete quality. To study the impact of sea water environment, pre-spawners of Atlantic salmon Salmo salar $\left(2^{+}\right)$were either reared in sea cages until final sexual maturation or transferred to fresh water in June or October. The beginning of spermiation or ovulation was recorded by weekly checking using hand-stripping. The quality of spawners was tested by fertilizing batches of 200 eggs, using DIA $532^{\circledR}$ insemination extender with sperm dilution of $10^{-3}$ and assessing survival at eyeing stage. Males transferred to fresh water (ED) presented normal spermiation cumulative frequency curves in both fresh water groups.

Females transferred to fresh water ovulated from November to December while ovulation of females reared in sea water was delayed and the period lengthened. Furthermore, only $67 \%$ of the EM females had ovulated during the experiment up to April. Relative fecundity of females transferred to ED in spring was significantly lower than that of females maintained in EM during summer. In spite of delayed and lengthened ovulation and of significantly higher osmolality of coelomic plasma $(348 \pm 13 \mathrm{mOsmol} / \mathrm{h}$, in EM and $270 \pm 23 \mathrm{mOsmol} / \mathrm{l}$ in ED) eggs of EM females were of a similar high quality to those of ED females, when fertilized less that one week after ovulation.

Males reared in sea water presented similar but slightly earlier spermiation curves. Heterogeneous and lower mean semen quality were recorded in EM males as compared with the ED males. Semen plasma osmolality was significantly higher in EM males $(347 \pm 27 \mathrm{mOsmol} / \mathrm{l}$ in $\mathrm{EM}$ and $265 \pm 30 \mathrm{mOsmol} / \mathrm{h}$ in ED). EM sperm that show low fertilizing capacity gave similarly poor results with insemination diluents at varying osmolalities (150 to $450 \mathrm{mOsmol} / \mathrm{l})$. Furthermore, no relationship could be established between seminal plasma osmolality and low fertility for each individual.

In terms of breeding management, seminal plasma osmolality cannot be retained as a criterion for selecting "good" EM males. Oocytes ageing in the abdominal cavity should be strictly avoided for females reared in sea water.

Keywords: Salmo salar, spermiation, ovulation, overmaturation, gamete quality, fertilization, osmoregulation, sea water, fresh water. 
Résumé

\section{INTRODUCTION}

Depuis le début de l'élevage du saumon atlantique, Salmo salar, la gestion de la reproduction des géniteurs implique généralement le retour des futurs reproducteurs en eau douce avant la ponte comme cela a été rapporté par Harache (1975) en Norvège. Il est intéressant pour des raisons pratiques, sanitaires et économiques, d'éviter cette manipulation. Aucune référence bibliographique n'aborde la description d'une optimisation technique de cette procédure ainsi que les effets induits sur les cinétiques de maturation sexuelle et sur la qualité des gamètes chez le saumon atlantique.

Chez plusieurs autres espèces de Salmonidés, le maintien en mer des animaux sexuellement matures se traduit par une altération des capacités à osmoréguler et/ou à se reproduire. L'analyse bibliographique souligne certains antagonismes entre le déroulement de la phase finale de la maturation sexuelle et le maintien en mer des géniteurs. Elle permet de regrouper les observations selon deux types caractérisant le déroulement de la maturation sexuelle et la qualité des gamètes produits.

Sower et Schreck (1982a et $b$ ) montrent qu'un maintien prolongé en mer de géniteurs de saumon coho, Oncorhynchus kisutch, induit de profonds déséquilibres osmotiques et endocriniens se traduisant par de fortes mortalités sans ovulation, des ovulations incomplètes et une diminution des réponses aux injections d'inducteurs de pontes efficaces chez les géniteurs ramenés en eau douce. Chez la même espèce et chez le saumon rose, O. gorbusha, Wertheimer (1984) n'observe aucun retard des ovulations et des spermiations alors que Lam et al. (1982, cité par
Wertheimer) observent un retard de 2 à 3 jours des ovulations. Harache et Faure (1986) soulignent des mortalités plus importantes des mâles maturant précocément de truite arc-en-ciel, O. mykiss, lors de leur transfert en mer. Thorpe (1986) note de fortes mortalités de tacons de saumon atlantique maturant accompagnées de pertes des capacités à osmoréguler. Quillet et al. (1992) rapportent que le maintien en mer des reproducteurs de truite fario, Salmo trutta fario, induit un retard et un étalement des ovulations ainsi que de fortes mortalités avant les pontes qui sont souvent incomplètes.

Wertheimer (1984) attribue la mauvaise qualité des croisements aux spermes et aux ovules chez le saumon coho et aux ovules chez le saumon rose. Chez les mêmes espèces Lam et al. (1982) observent une altération de la qualité des gamètes mâles.

De nombreux auteurs ont confirmé l'existence d'une osmolarité anormalement importante des fluides plasmatiques, séminaux et ovariens chez de nombreuses espèces de Salmonidés lorsqu'elles sont maintenues en mer lors de la reproduction (Kubo, 1960, cité par Fontaine, 1975; Hirano et al., 1978 et Morisawa et al., 1979; Sower et Schreck, $1982 a$ et Wertheimer, 1984).

Ces résultats permettent de supposer des problèmes relatifs à l'efficacité de reproduction des géniteurs maintenus en eau de mer dues à des interactions physiologiques entre les fonctions de reproduction et d'osmorégulation.

La présente étude vise à évaluer les capacités de reproduction de géniteurs de saumon atlantique élevés puis maintenus en mer pendant la reproduction par rapport à des géniteurs transférés en eau douce avant le début de l'ovulation des femelles. Pour cela, les 
cinétiques d'entrée en ovulation et en spermiation sont décrites; les qualités des gamètes mâles et femelles sont «testées » par des fécondations intraet inter-traitements par analyse des pourcentages d'embryonnement au stade oeillé; enfin, l'osmolarité des liquides séminaux et coelomiques est mesurée et comparée dans les différents groupes expérimentaux.

Nous avons comparé les performances de reproduction de géniteurs maintenus en mer (EM) ou transférés en eau douce (ED) à 2 périodes différentes de la gamétogenèse :

- en juin, pour étudier l'influence d'un transfert précoce en eau douce sur l'ensemble de la gamétogenèse (EDP);

- en octobre, pour étudier l'influence d'un transfert tardif en eau douce sur les processus finaux de maturation (EDT).

L'osmolarité en moyenne plus élevée des liquides séminaux et coelomiques des animaux maintenus en mer apparaissant associée à la baisse de qualité des gamètes, nous étudions la relation entre l'osmolarité du plasma séminal et le pouvoir fécondant individuel des spermes; enfin, pour évaluer le rôle potentiel du gradient osmotique subi par les spermatozoïdes EM lors de leur mise en contact avec le dilueur de fécondation utilisé en routine et ajusté à $250 \mathrm{mOsmol} / \mathrm{l}$, l'influence de l'osmolarité du dilueur sur le pouvoir fécondant des spermes est étudiée.

\section{MATÉRIEL ET MÉTHODES}

\section{Les géniteurs}

Les trois lots expérimentaux étaient réalisés à partir de reproducteurs $\left(2^{+}\right)$de la souche de la rivière Elorn (Bretagne) de deuxième génération de domestication. Les futurs reproducteurs ont successivement été élevés en eau douce à la station IFREMER du Conquet puis transférés au stade smolt dans des cages en mer $(35 \%)$ de $120 \mathrm{~m}^{3}$ à la Société SEMII à Camaret.

Trois lots de géniteurs âgés de $2^{+}$ans ont été constitués. Le premier lot a été maintenu en mer jusqu'à la fin de l'expérience (lot EM) en cage de $60 \mathrm{~m}^{3}$ au large de Camaret à la SEMII.

Le deuxième lot de 360 animaux EDP (lot Eau Douce transfert Précoce) a été constitué à partir du lot EM, le 16 juin 1987, et transféré en eau douce dans des bassins circulaires de $25 \mathrm{~m}^{3}$ à la charge de $5 \mathrm{~kg} / \mathrm{m}^{3}$ au Centre IFREMER de Brest. La salinité de l'eau des bassins a été ramenée à $20 \%$, le 17 , à $10 \%$ le 18 et à $0 \%$ le 22 juin. 10 animaux sont morts entre le transfert et le 8 juillet et 6 sont morts entre cette date et le 7 octobre soit $4,4 \%$ des 360 animaux initiaux.

Les 3,4 et 5 octobre 1987 , un tri sur des critères morphologiques sur ces deux premiers lots a permis de ne conserver que des animaux devant arriver à maturité durant l'hiver. À ces mêmes dates, le troisième lot a été constitué à partir du lot EM trié. Ce lot a été transféré en eau douce, au Centre IFREMER le 7 octobre, dans les mêmes conditions que le lot EDP. Il constitue le lot EDT (Eau Douce Tardif). 3 sujets sont morts le 16 décembre.

$\mathrm{Au}$ début de l'expérimentation, les effectifs de mâle et de femelle des 3 lots EM, EDP et EDT, établis d'après des critères morphologiques, étaient respectivement de 81 et 73,81 et 80 et 82 et 58 .

\section{Performances de reproduction}

Les entrées en spermiation et les ovulations de la totalité des 463 géniteurs en expérimentation ont été suivies, selon une fréquence hebdomadaire, par pression abdominale après anesthésie au phénoxyéthanol ( $3 \mathrm{ml} / 101$ d'eau additionnée de furoxone) d'octobre 1987 à avril 1988. 231 fécondations ont été réalisées en trois séries, du fait de l'étalement des ovulations, les 29 décembre ( 72 fécondations), 12 janvier (45 fécondations) et 4 février (114 fécondations). Les spermes provenaient de mâles spermiants depuis respectivement 5,8 et 11 semaines. Les inséminations ont été réalisées suivant la méthode décrite par Billard (1977) modifiéc par Maisse et Porcher (1981) à partir de $20 \mathrm{~g}$ d'ovules fécondés par $100 \mu \mathrm{l}$ de sperme dans $100 \mathrm{ml}$ de dilueur de fécondation DIA $532^{\circledR}$ préconisé par Billard (1977).

Les pontes de femelles ovulées depuis moins d'une semaine ou depuis une à deux semaines ont été utilisées. L'effet du vieillissement post-ovulatoire dans la cavité abdominale sur la qualité des ovules a été étudié au cours d'une expérience complémentaire réalisée les 11,19 et 26 janvier sur 11 femelles du lot EDT, (repérées individuellement avec des marques «Floy tag ») en utilisant un mélange de spermes de 5 mâles du même lot. La viabilité des œufs obtenus a été estimée par complage des embryons survivants au stade œillé.

\section{Analyse des caractéristiques du sperme}

L'osmolarité des liquides coelomiques et séminaux a été évaluée le 3 février sur les surnageants à la précision de $\pm 2 \mathrm{mOsmol} / \mathrm{l}$ après centrifugation des spermes à $250 \mathrm{~g}$ pendant 20 minutes.

En 1990, la relation entre l'osmolarité individuelle de chaque sperme et la fécondance était évaluée sur des animaux de la même population $\left(2^{+}\right)$maintenus en mer jusqu'à la collecte de la laitance (lot EM) ou transférés en eau douce fin octobre (lot EDT). Le jour de l'expérience (fin décembre), chaque sperme était récolté et centrifugé pour mesurer la pression osmotique du liquide séminal suivant le protocole déjà décrit. Nous avons choisi 8 mâles EM et 8 mâles ED dont les pressions osmotiques individuelles des liquides séminaux variaient entre 194 et $300 \mathrm{mOsmol} / \mathrm{l}$ pour les mâles ramenés en eau douce en octobre et entre 304 et $417 \mathrm{mOsmol} / \mathrm{l}$ pour ceux laissés en mer, afin d'établir une éventuelle relation entre ce 
paramètre et la fertilité des spermes. La qualité des différents spermes a été " testée " sur 7 aliquotes de chaque culot de sperme comme décrit plus haut, mais en utilisant 7 dilueurs de fécondation différents dont les osmolarités étaient fixées de 150 à $450 \mathrm{mOsmol} / \mathrm{l}$ en faisant varier la concentration en $\mathrm{NaCl}$ de chaque dilueur. Les fécondations ont été effectuées avec un lot d'œufs provenant de 5 femelles ramenées en eau douce à la même époque que les mâles.

\section{Analyse statistique}

Les écarts aux moyennes sont exprimés par les erreurs standard à $p<0,05$. Les pressions osmotiques des fluides séminaux ont été analysées par test de $t$ de Student à $p<0,05$ et celles des fluides ovariens par ANOVA à $p<0,01$ après vérification de l'homogénéité des variances. Les poids des femelles ovulées ont été testés par test de Kuskal et Wallis à $p<0,05$. Les fécondités totales ont été testées par ANOVA $(p<0,01)$ après vérification de l'homogénéité des variances et les fécondités relatives par test de Kruskal et Wallis à $p<0,05$. Les poids individuels des œufs à la ponte ont été testées par test de Kruskal et Wallis à $p<0,05$.

Du fait du faible nombre de femelles synchrones, de la répartition non-normale des données de fécondation, même après transformation, et de l'asymétrie des plans de fécondations croisées, les qualités des gamètes ont été analysées par Analyse factorielle des Correspondances à partir du tableau de contingence réalisé en répartissant les différents résultats de chaque type de croisement suivant 5 catégories de qualité de 0 à $20 \%$, de 20 à $40 \%$, de 40 à $60 \%$, de 60 à $80 \%$ et de 80 à $100 \%$ d'embryonnement.

L'effet des différentes pressions osmotiques des dilueurs sur les taux d'embryonnement a été évaluée par ANOVA à $p<0,01$ après vérification de l'homogénéité des variances et l'effet de la pression osmotique initiale des spermes des animaux maintenus en mer sur les taux d'embryonnement a été évalué par test de Kruskal et Wallis à $p<0,05$.

\section{RÉSULTATS}

Durant l'été précédent la ponte, les températures ont été en moyenne de $13^{\circ} \mathrm{C}$ en eau douce et de $16^{\circ} \mathrm{C}$ en mer avec des maxima à $17,5^{\circ} \mathrm{C}$. Du 17 juin au 10 octobre, la température est en moyenne plus élevée de $1,5^{\circ} \mathrm{C}$ en mer qu'en eau douce. Elle est ensuite sensiblement de la même valeur de $7,2^{\circ} \mathrm{C}$ en moyenne à partir de la mi-décembre.

Les températures observées en mer en 1987 et 1988 sont supérieures de l'ordre de $+1^{\circ} \mathrm{C}$ lors de la période estivale et de +2 à $3^{\circ} \mathrm{C}$ lors de la période hivernale par rapport à la moyenne des années 1985-1986 et 1987 et sensiblement équivalentes du 15 septembre au 15 décembre.

Les animaux ont cessé de s'alimenter dès leur retour en eau douce, contrairement à ceux restés en mer, et malgré une distribution minimale journalière d'aliment de $0,5 \%$ du poids vif.

\section{Cinétique de spermiation et d'ovulation ( $f$ ig. 1)}

Les lots maintenus en mer durant l'été, EDT et $\mathrm{EM}$, présentent une légère avance des entrées en spermiation. L'extrapolation des courbes d'entrée en spermiation en fonction du temps permet d'évaluer le début de celles-ci vers le 20 septembre 1987 pour les lots EM et EDT et 20 jours après, soit le 10 octobre, pour le lot EDP (cette avance se réduit cependant pour le lot EDT qui atteint les $100 \%$ de mâles spermiants en même temps que le lot EDP).

Les médianes ( $50 \%$ de mâles spermiants) des 3 lots, plus objectives car réellement mesurées, sont atteintes respectivement le 30 octobre (EM), le 7 novembre (EDT) et le 20 novembre (EDP). Les entrées en spermation s'étalent sur 2,5 mois et présentent des cinétiques de déroulement globalement peu différentes. À l'arrêt du tri hebdomadaire, le 4 février 1988 en eau douce, $84 \%$ des mâles étaient encore spermiants et $67 \%$ présentaient plus de 11 semaines de spermiation. Le 6 avril, $45 \%$ des mâles EM étaient encore spermiants et $60 \%$ présentaient une durée de spermiation supérieure à 17 semaines.

Les femelles des lots transférés en eau douce, EDP et EDT, commencent à ovuler le 8 décembre alors que celles du lot EM ne débutent que 15 jours plus tard, le 23 décembre. Les pentes des courbes de pourcentages d'ovulation cumulés en fonction du temps sont similaires pour les deux lots transférés en eau douce alors que celle du lot resté en mer est nettement plus faible. Les $50 \%$ d'ovulation sont atteints respectivement le 28 décembre (EDT), le 10 janvier (EDP) et le 3 mars (EM). Les ovulations se répartissent sur 2 mois pour les lots ramenés en eau douce et sur plus de 3,5 mois pour les femelles maintenues en mer. À la fin de l'expérience, $33 \%$ des femelles de ce dernier lot n'avaient pas encore ovulé.

\section{Fécondité}

La taille des œufs à la ponte n'est pas significativement différente entre les 3 origines. La fécondité totale des femelles transférées tardivement en eau douce est significativement plus élevée (2817 œufs $\pm 501 ; n=10$ ) que celle des femelles maintenues en mer $(2174 \pm 296 ; n=17)$ ou transférés au printemps $(2409 \pm 298 ; n=29)$. Les femelles du lot EM retenues pour les fécondations ( $22 \%$ des femelles du lot) sont de poids significativement inférieur (1140 $\pm 128 \mathrm{~g})$ à celles des deux autres lots qui ne differrent pas significativement entre elles (1 506 $\pm 113 \mathrm{~g} / \mathrm{EDP}$ et $1434 \pm 162 \mathrm{~g} / \mathrm{EDT})$. La fécondité relative des femelles transférées en juin (1608 cufs $/ \mathrm{kg} \pm 159$ ) est significativement inférieure aux fécondités relatives des femelles maintenues en mer $(1890 \pm 210)$ ou transférées en octobre 


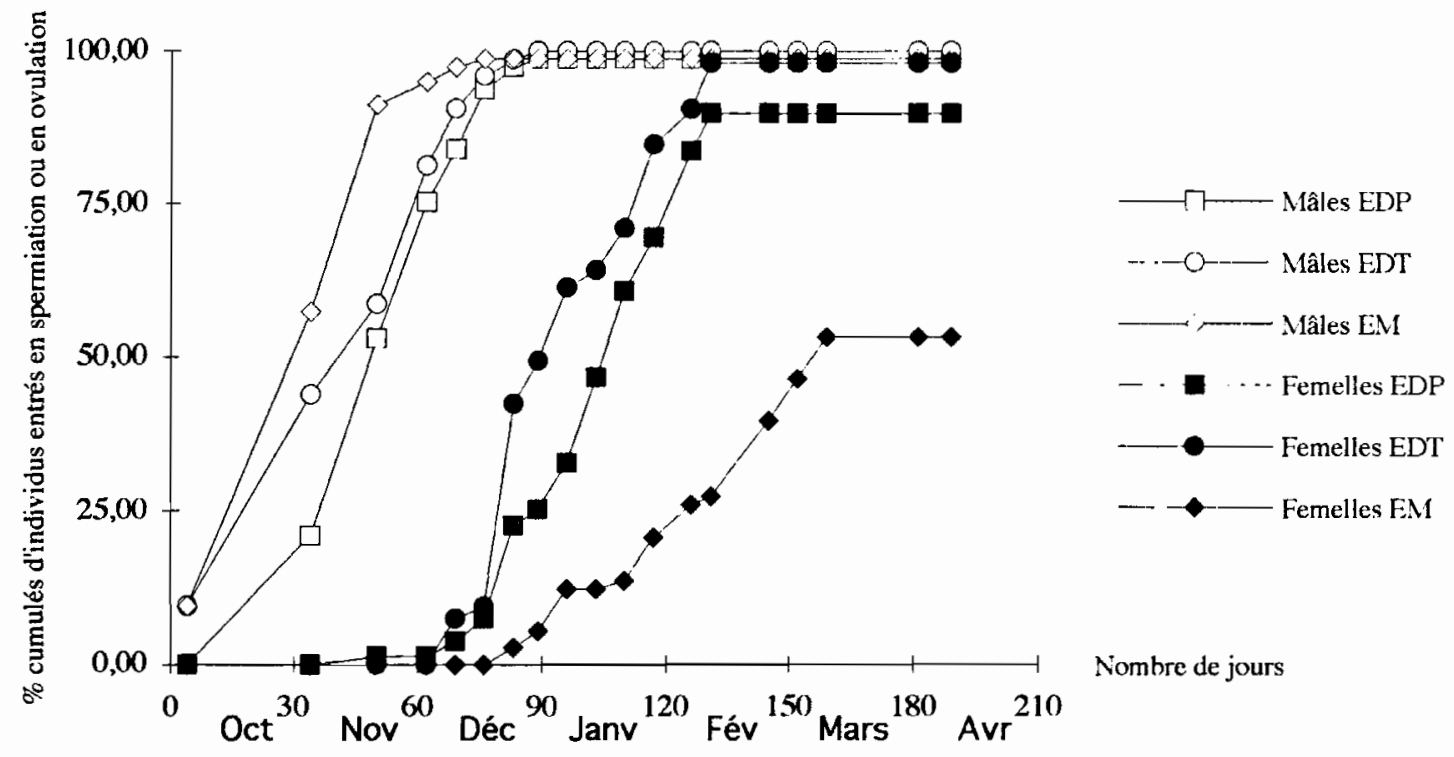

Figure 1. - Profils cumulés d'entrées en spermiation et en ovulation de trois lots de saumon atlantique $\left(2^{+}\right)$ramenés précocément en juin (EDP) ou tardivement en eau douce en octobre (EDT) ou maintenus en mer jusqu'à la ponte (EM).

Cumulative ovulation and spermiation profiles of Atlantic salmon $\left(2^{+}\right)$transferred into fresh water in June (EDP), in October (EDT) or held in sea water (EM) until final sexual maturation.

$(1932 \pm 210)$. Les deux derniers groupes ne diffèrent pas significativement entre eux.

\section{Qualité des gamètes}

Dans l'étude préliminaire menée sur des femelles EDT, la cinétique de vieillissement des ovules laissés dans la cavité abdominale 1 à 3 semaines après l'ovulation montre une baisse progressive de leur qualité qui se traduit comme suit: le taux d'embryonnement individuel varie entre 77 et $99 \%$ la première semaine, de 31 à $95 \%$ la seconde et de 0 à $67 \%$ la troisième; le taux d'embryonnement moyen diminue de $92 \%$ la première semaine à $75 \%$ la seconde et à $31,5 \%$ la troisième. Dans la suité des résultats, du fait de ces différences marquées, les données obtenues avec des ovules âgés de moins d'une semaine ou d'une à deux semaines sont analysées séparément.

Les données de fécondation sont rassemblées figure 2.

Les croisements réalisés entre les mâles ramenés en eau douce et les femelles des mêmes lots ou maintenues en mer et ovulées depuis moins d'une semaine correspondent à des taux d'embryonnement élevés $(>80 \%)$. Lorsque ces mêmes femelles sont croisées avec des mâles maintenus en mer, les moyennes des taux d'embryonnement chutent de 15 à $40 \%$ et la variabilité intra-lot augmente de façon importante (30\% en moyenne). Cependant il faut remarquer que certains mâles EM présentent individuellement de très bon taux de fécondation. Ainsi, 57\% des spermes EM utilisés permettent d'obtenir des taux d'embryonnement supérieurs à $60 \%$ lorsque ces géniteurs sont croisés avec des femelles ramenées en eau douce précocément (EDP).

La qualité des cufs des femelles ramenées en eau douce à l'automne, EDT, n'apparaît pas différente de celle du lot ramené au printemps EDP, quelle que soit la durée de l'intervalle depuis l'ovulation, ni de celles maintenues en mer lorsque les femelles sont utilisées la première semaine après l'ovulation. Ce taux moyen d'embryonnement tombe lorsque les ovules des femelles maintenues en mer sont utilisés entre 1 à 2 semaines après l'ovulation. Cette chute est encore amplifiée lorsque des mâles EM sont utilisés $(28,2 \%)$.

La projection à partir du tableau de contingence 1 sur les deux premiers axes de l'analyse factorielle des correspondances ( $f$ ig. 3 ) exprime $90,1 \%$ de l'inertie totale et constitue une très bonne représentation des relations entre les classes de fécondation. Certains croisements bien exprimés dans ce plan de projection (soit ceux dont la somme des cosinus carrés des coordonnées est supérieure à 0,6 ) peuvent être associés avec des classes de fécondation.

- Les croisements $\mathrm{MM}_{2}, \mathrm{MT}_{2}$ et $\mathrm{MP}_{2}$ réalisés avec des mâles maintenus en mer $M$ et des femelles utilisées 1 à 2 semaines après l'ovulation $\left(\mathbf{M}_{2}\right.$, $\mathrm{T}_{2}, \mathrm{P}_{2}$ ) sont principalement associés à des classes d'embryonnement compris entre 0 à $40 \%$.

- Les croisements $\mathrm{TM}_{2}, \mathrm{PM}_{2}$ et $\mathrm{MP}_{1}$ réalisés avec des mâles transférés en eau douce ( $\mathrm{P}$ et $\mathrm{T}$ ) avec des femelles maintenues en mer et de plus d'une semaine d'ovulation $\left(\mathrm{M}_{2}\right)$ ou entre mâles maintenus en mer $(\mathrm{M})$ 


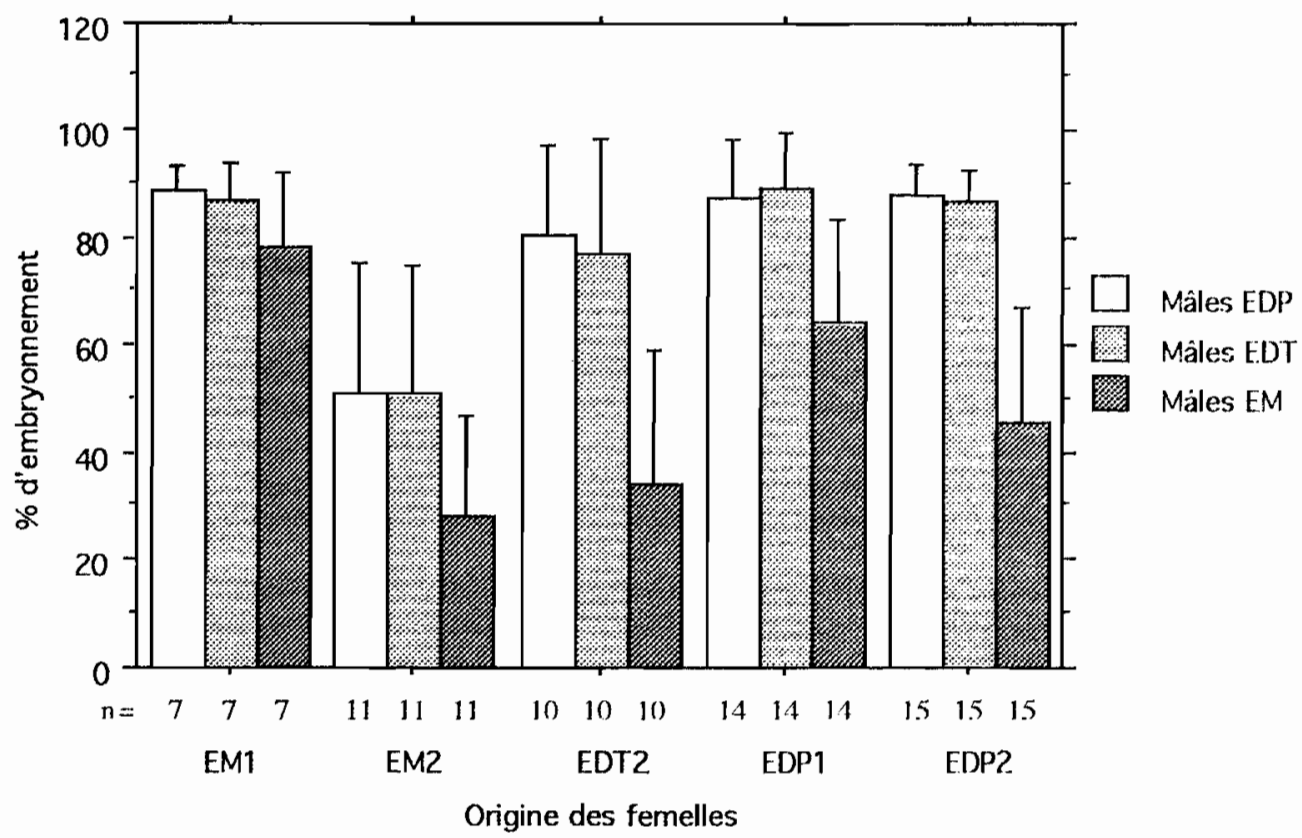

Figure 2. - La qualité des gamètes des différents types de croisements a été mesurée par le \% d'ceufs embryonnés au stade œillé, après fertilisation de lots de 200 ceufs par $100 \mu \mathrm{l}$ de sperme et $100 \mathrm{ml}$ de DIA 532\% . Chaque barre représente la moyenne des taux d'embryonnement \pm intervalle de confiance $(p<0,05)$ des 15 croisements individuels entre les mâles et les femelles transférés en eau douce en juin (P pour précoce), en octobre ( $T$ comme tardif) ou maintenus en mer ( $M$ pour mer) jusqu'à la reproduction. $M$ ou $F$ représentent le sexe des géniteurs des croisements ( $M=$ mâle et $F=$ femelles) $P, T$ et $M$ représentent leur milieu de maturation finale $(P=$ transfert précoce en juin, $T=$ transfert tardif en octobre et $M=$ maintien en mer). Les chiffres 1 ou 2 représentent le nombre de semaine entre la date d'ovulation présumée et la fécondation.

Gamete quality of the different crosses was assessed by measuring the percentage of survival to eyeing stage, after fertilization of 200 eggs with $100 \mu \mathrm{l}$ of sperm in $100 \mathrm{ml}$ of DIA532 $2^{\mathfrak{B}}$. Each bar shows the mean \pm confidence interval $(p<0,05)$ of the 15 cross-fertilization between males and females transferred into fresh water in June ( $P$ for "precoce") or in October ( $T$ for "tardif") or maintained in sea water ( $M$ for "mer") until reproduction. The first letter $(P, T, M)$ stands for the male origin, the second letter $(P, T, M)$ for the female origin and the number 1 or 2 stands for the number of weeks between ovulation and female fertilization.

avec des femelles transférées en eau douce de moins d'une semaine d'ovulation $P_{1}$ sont surtout associés à des classes d'embryonnement de 40 à $60 \%$.

- Le croisement $\mathrm{MM}_{1}$ réalisé avec des mâles $\mathbf{M}$ et des femelles maintenues en mer de moins d'une semaine d'ovulation $\mathrm{M}_{1}$ tend à être associé à la classe d'embryonnement de 60 à $80 \%$.

- Les croisements $\mathrm{PP}_{1}, \mathrm{TP}_{1}, \mathrm{TM}_{1}, \mathrm{PP}_{2}$ et $\mathrm{TT}_{2}$ réalisés avec des mâles $P$ et $T$ et des femelles transférées en eau douce puis utilisées 2 semaines après l'ovulation $\left(\mathrm{P}_{1}, \mathrm{P}_{2}, \mathrm{~T}_{2}\right)$ ou maintenues en mer

Tableau 1. - Tableau de contingence des différents types de fécondation dont les performances sont répartis en 5 classes de qualité suivant le taux d'embryonnement au stade ceillé. M:Eau de mer (EM), P: Eau Douce Précoce (EDP) et T:Eau Douce Tardif (EDT). Les chiffres 1 et 2 représentent des ovules de femelles de moins d'une ou entre une et deux semaines après l'ovulation.

Correspondent Factorial Analysis table. The different crosses were subdivided in 5 classes of quality from the \% of survival at eyeing stage. The letters on the first line $(P, T, M)$ stand for the male origin, on the second line $(P, T, M)$ for the female origin and the number 1 or 2 stands for the number of weeks between ovulation and female fertilization ( $P$ : fish transferred into fresh water in June, T: in October, M: fish held in sea water).

\begin{tabular}{|c|c|c|c|c|c|c|c|c|c|c|c|c|c|c|c|}
\hline \multirow{2}{*}{$\begin{array}{l}\text { Mâles } \\
\text { Femelles }\end{array}$} & \multicolumn{5}{|c|}{$\begin{array}{c}\text { Eau douce } \\
\mathrm{T}\end{array}$} & \multicolumn{5}{|c|}{$\begin{array}{c}\text { Eau de mer } \\
M\end{array}$} & \multicolumn{5}{|c|}{$\begin{array}{c}\text { Eau douce } \\
P\end{array}$} \\
\hline & $M_{1}$ & $\mathrm{M}_{2}$ & $\mathrm{~T}_{2}$ & $P_{1}$ & $\mathrm{P}_{2}$ & $\mathrm{M}_{1}$ & $\mathrm{M}_{2}$ & $\mathrm{~T}_{2}$ & $P_{1}$ & $\mathrm{P}_{2}$ & $\mathrm{M}_{1}$ & $\mathrm{M}_{2}$ & $\mathrm{~T}_{2}$ & $P_{1}$ & $\mathrm{P}_{2}$ \\
\hline $0-20 \%$ & 0 & 3 & 1 & 0 & 0 & 0 & 4 & 4 & 2 & 5 & 0 & 3 & 0 & 0 & 0 \\
\hline $20-40 \%$ & 0 & 1 & 1 & 1 & 0 & 0 & 5 & 3 & 1 & 3 & 0 & 1 & 1 & 0 & 0 \\
\hline $40-60 \%$ & 0 & 1 & 0 & 0 & 0 & 1 & 1 & 1 & 3 & 2 & 0 & 2 & 1 & 2 & 1 \\
\hline $60-80 \%$ & 1 & 3 & 0 & 1 & 4 & 2 & 0 & 1 & 2 & 0 & 1 & 2 & 0 & 1 & 1 \\
\hline Total & 6 & 12 & 10 & 14 & 15 & 6 & 12 & 10 & 14 & 15 & 6 & 12 & 10 & 14 & 15 \\
\hline
\end{tabular}




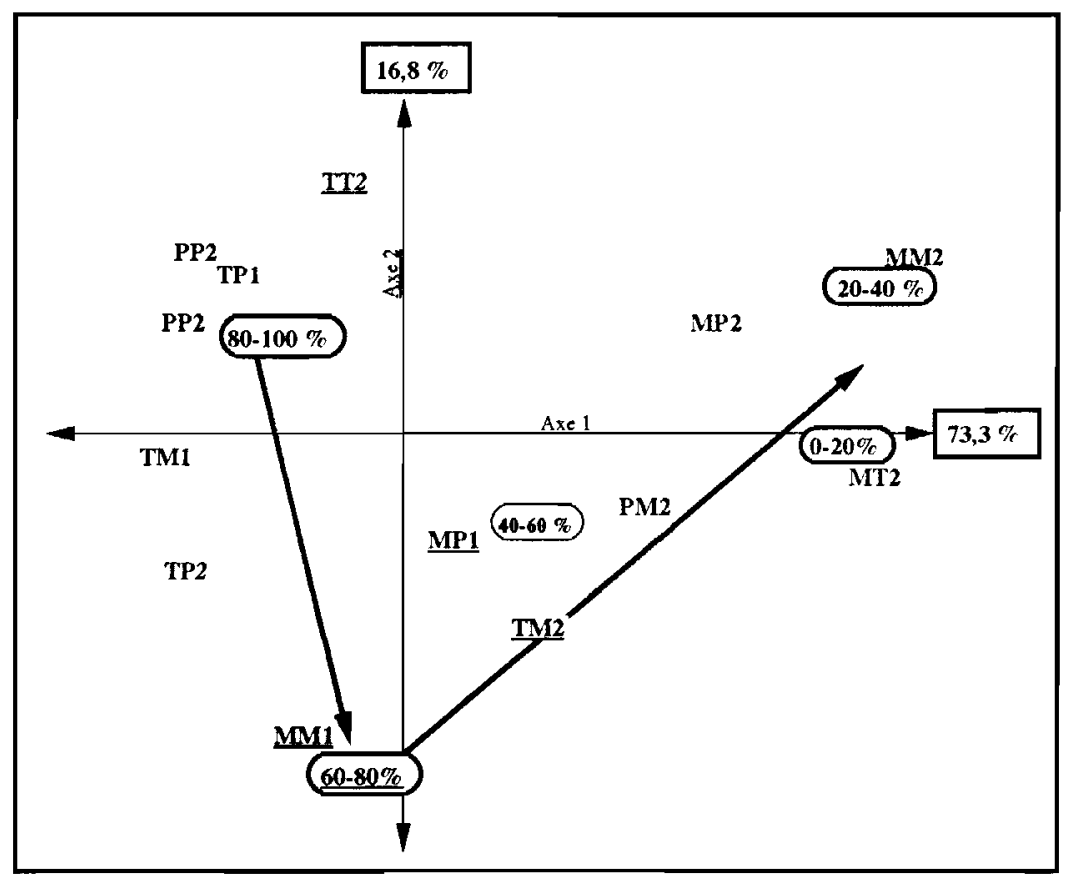

Figure 3. - Projection de l'Analyse Factorielle des Correspondances sur les axes 1 et 2 (pourcentages des variations expliquées par les axes en encadrés rectangulaires) du tableau de contingence 1 (voir texte) des différents types de croisements bien représenté (cos $\left.{ }^{2}>0,6\right)$ et de classes de qualité (encadrées en ovale) des fécondations au stade cillé entre des mâles et des femelles de saumon atlantique $\left(2^{+}\right)$ramenés précocément ou tardivement en eau douce en juin ou en octobre ( $\mathrm{P}, \mathrm{T})$ ou maintenus en mer jusqu'à la maturation sexuelle $(\mathrm{M})$. La première lettre représente l'origine du mâle, la deuxième celle des femelles et les chiffres 1 ou 2 correspondent aux femelles ayant respectivement ovulé depuis moins d'une semaine (1) ou entre une et deux semaines (2). Les croisements et les classes de qualité bien représentés sur l'axe 2 sont soulignés. La première lettre $(P, T, M)$ représente l'origine du mâle, le deuxième $(P, T, M)$ celle des femelles et les chiffres 1 ou 2 représentent le nombre de semaine entre la date d'ovulation présumée et la fécondation.

Correspondent Factorial Analysis projection of the interaction between fertilization crosses and quality classes of fertilization obtained with Atlantic salmon held in sea water $(M)$ until reproduction or transferred in fresh water in June $(P)$ or in October $(T)$ taken from table 1 . The percentage of the variation explained by the axe is shown in by black framed squares. The quality of the different classes is shown in the oval frame. Classes and crosses with good representation on Axe 1 are in bold type and those with good representation on the Axe 2 are underlined. The first letter $(P, T, M)$ stands for the male-origin, the second letter $(P, T, M)$ for the female origin and the number 1 or 2 stands for the number of week between ovulation and female fertilization.

et ovulées depuis moins d'une semaine $\left(\mathrm{M}_{1}\right)$ sont associés à la classe de 80 à $100 \%$.

\section{Osmolarité des fluides génitaux et effet des dilueurs de fécondation}

Lors de la première partie de l'expérience en 1987, l'osmolarité des liquides ovariens des femelles ramenées en eau douce avant l'été EDP $(270 \pm 14 \mathrm{mOsmol} / 1 ; n=9)$ et des liquides séminaux des mâles du même lot et du lot EDT $(265 \pm 8 \mathrm{mOsmol} / \mathrm{l}$ avec $n=8 ; 265 \pm 10 \mathrm{mOsmol} / \mathrm{l}$ avec $n=8$ ) n'est pas significativement différente entre les lots. Par contre, l'osmolarité des liquides ovariens et séminaux des géniteurs maintenus en mer est significativement plus élevés $(348 \pm 11 \mathrm{mOsmol} / 1$ avec $n=9$ pour les femelles et $347 \pm 10 \mathrm{mOsmol} / \mathrm{l}$ avec $n=8$ pour les mâles), mais identique entre les sexes.

En 1990, les moyennes des survies au stade œillé obtenues avec les spermes des mâles maintenus en mer étaient généralement inférieures à celles obtenues avec les mâles ramenés en eau douce. Toutefois, aucune corrélation significative n'a pu être mise en évidence entre l'osmolarité des plasma séminaux et le taux d'embryonnement individuel obtenu avec les spermes correspondants que l'on fasse l'analyse sur l'ensemble des fécondations, sur les sous-ensembles mâles EM et ED séparément, ou pour un dilueur de fécondation donné.

On observe un effet hautement significatif de l'osmolarité du dilueur sur les performances des spermes des populations EM et ED (fig. 4): l'osmolarité la plus favorable pour les spermes des animaux ramenés en eau douce se situe entre 250 et $300 \mathrm{mOsmol} / \mathrm{l}$ alors que pour ceux laissés en mer elle se situe entre 250 et $350 \mathrm{mOsmol} / 1$. Les conditions extrêmes de pressions osmotiques des dilueurs, 150 et $450 \mathrm{mOsmol} / \mathrm{l}$, réduisent très fortement les taux d'embryonnement, quelle que soit l'origine des spermes. 


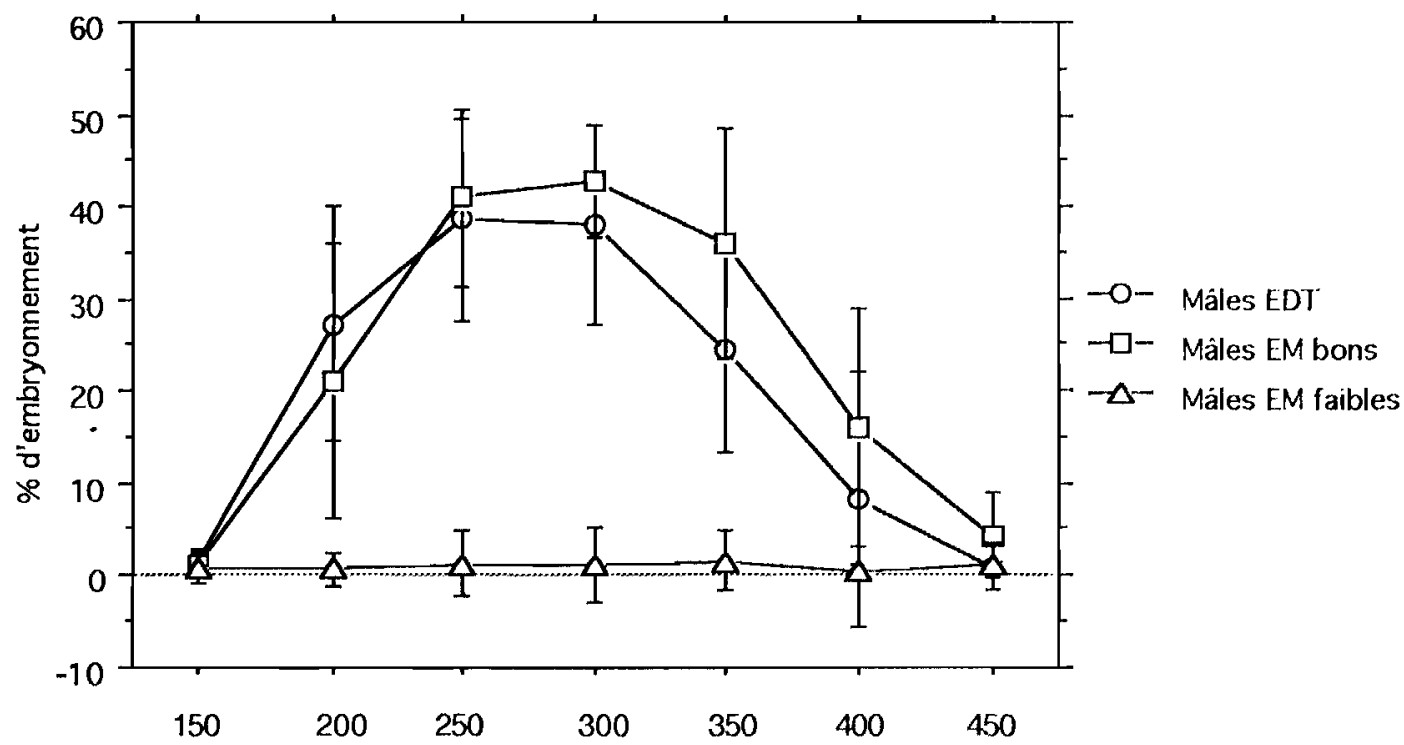

Pression osmotique des dilueurs expérimentaux en mOsmol $/$

Figure 4. - Pourcentage d'embryonnement au stade ceillé de fécondations entre un pool d'ovules de femelles de saumon atlantique $\left(2^{+}\right)$ et de mâles ramenés tardivement en eau douce (EDT) ou de mâles maintenus en mer (EM) avec différents dilueurs de fécondation de pression osmotiques entre 150 à $450 \mathrm{mOsmol}$. Les performances des mâles EM ont été présentés sous deux formes : la valeur moyenne des 8 testés, celle des 5 "bons" et celle des 3 « mauvais".

Effect of fertilization diluant osmolality on the fertilizing capacity of the sperm: the rate of survival at eyeing stage was measured after fertilization using extenders at different osmolality (150 to $450 \mathrm{mOsmol} / \mathrm{h})$, with individual sperm from males transferred to fresh water $(E D ; n=8)$ or maintained in sea water $(E M ; n=8)$. The results obtained with EM males are shown as the mean of 8 animals or the means are shown for the 5 good males ("bons") and the 3 bad males ("mauvais").

Les 8 spermes EM pourraient être subdivisés en 2 sous-populations ( $f$ ig. 4) : l'une constituée de spermes de bonne qualité $(n=5)$, qui induisent des taux de fécondation équivalents ou meilleurs que ceux obtenus avec les mâles ED; l'autre constituée des mâles qui donnent de mauvais résultats quelle que soit la pression osmotique testée $(n=3)$.

On peut en outre remarquer que les bons spermes des sujets maintenus en mer résistent significativement mieux aux fortes pressions osmotiques des dilueurs que les spermes ED $(36 \pm 6 \%$ contre $25 \pm 5 \%$ à $350 \mathrm{mOsmol} / \mathrm{l}, 16 \pm 5$ contre $2,8 \pm 1,3 \%$ à $400 \mathrm{mOsmol} / \mathrm{h}$ et $4 \pm 1$ contre $1 \pm 0,2 \%$ à $450 \mathrm{mOsmol} / 1)$.

\section{DISCUSSION}

Contrairement à ce qui est observé chez les saumons du Pacifique et la truite fario, aucune mortalité avant ou pendant la ponte n'est observée en mer lors de l'expérimentation. Les transferts précoces et tardifs n'ont induit qu'une faible mortalité, inférieure à $4,5 \%$. Les mesures d'osmolarité effectuées lors de ce travail sont en accord avec celles réalisées chez d'autres Salmonidés lors de la maturation finale en mer qui s'accompagne d'une élévation de la pression osmotique des liquides plasmatiques, séminaux et ovariens (Kubo, 1960, cité par Fontaine, 1975; Sower et Schreck, $1982 a$ et Wertheimer, 1984) et d'une chute et d'une stabilisation de ces mêmes paramètres trois jours après le transfert en eau douce (Hirano et al., 1978 et Morisawa et al., 1979).

\section{Cinétique de spermiation}

L'analogie des pentes des courbes d'entrées en spermiation entre les lots suggère que les cinétiques de maturation finale sont similaires chez les mâles et que les différences observées pourraient être dues aux différences mineures de températures entre l'eau douce et l'eau de mer durant l'été et durant la maturation finale (Billard et Breton, 1977).

\section{Cinétique d'ovulation}

L'environnement aquatique de stabulation pendant l'été n'induit pas de différence marquée entre les cinétiques de gamétogenèse des femelles transférées en eau douce, 6 mois ou 2 mois avant les premières ovulations. Cependant, un retard, un étalement important, voire un blocage des pontes sont observés si les femelles sont maintenues en mer jusqu'à l'ovulation, ce qui souligne l'effet néfaste de l'environnement marin sur les phases ultimes de la maturation ovocytaire et/ou sur les mécanismes de 
l'ovulation. Ce retard est à rapprocher de résultats précédents où les élévations des niveaux plasmatiques d'E2 et de GtH présentaient un retard de plusieurs semaines che $z$ les femelles maintenues en mer (Le Gac et al., 1986).

Ce résultat pourrait résulter : soit en un ralentissement de la vitellogenèse et/ou de la maturation finale dû au retard de l'élévation d'œstradiol et/ou de $\mathrm{GtH}$ ou soit d'une désensibilisation ovarienne à la GtH en milieu marin, comme cela a été suggéré par Sower et Schreck (1982b), chez le saumon coho.

Il semble que l'on puisse cependant y remédier puisque O'Flynn et al. (1992) et Zohar (1993) rapportent une relative synchronisation des ovulations en eau de mer par Ovaprim ${ }^{(3)}$ et un avancement et une synchronisation des ovulations avec des implants Reproboost ${ }^{(-)}$sur des saumons atlantique et des saumons coho maintenus en mer lors de la ponte. Cependant une qualité moyenne de gamètes $(70 \%$ d'embryonnement), inférieure à celle observée dans notre expérimentation est rapportée par les deux auteurs.

Par ailleurs, on ne peut exclure qu'une perturbation de la maturation folliculaire et de l'hydratation des ovules précédant l'ovulation (pouvant représenter jusqu'à $15 \%$ du poids des ovules) soit due à l'osmolarité élevée des liquides physiologiques en mer.

\section{Fécondité}

Les poids moyens d'un cuf ne sont pas significativement différents entre les lots à la ponte. Il ne peut cependant être exclu qu'à la fin du développement embryonnaire une différence significative s'établisse suivant le milieu final de l'ovulation.

Les femelles transférées en juin présentent une fécondité relative plus faible que celles maintenues en mer durant la période estivale et ramenées en eau douce avant l'hiver alors que leurs poids sont similaires. Plusieurs auteurs ont montré que le recrutement des ovocytes en vitellogenèse active pouvait être altéré par le manque de réserve métabolique disponible chez le saumon rose (Persov, 1963 ; Melnikova, 1964 in Prouzet et Gaignon, 1982) et Le Bail (1984) a souligné que, chez le saumon atlantique sauvage, la saison de migration en eau douce pouvait influer sur le nombre d'œufs recrutés en vitellogenèse. Dans notre expérience, le transfert précoce a été effectué en juin, lors du recrutement des ovocytes en vitellogenèse active et a été suivi d'un arrêt de prise alimentaire en eau douce. Une interaction entre les nouvelles conditions d'osmorégulations et les niveaux endocriniens conditionnant le déroulement de la maturation sexuelle ne peut être exclue.

La fécondité absolue plus basse des femelles maintenues en mer s'explique par le poids plus faible des femelles ayant ovulé.

\section{Qualité des gamètes}

Des différences importantes de qualité d'embryonnement ont été mises en évidence selon le type de croisements et de géniteurs. En ce qui concerne les gamètes femelles, les deux traitements, aboutissant à un retour des génitrices en eau douce, procurent des ovules de très bonne qualité. Un effet modeste du maintien en mer a été observé lorsque les ovules sont collectés moins d'une semaine après l'ovulation. Par contre, la baisse de qualité due au vieillissement paraît considérablement accentuée lorsque les ovules sont maintenus dans la cavité abdominale plus d'une semaine après l'ovulation en mer (soit ici après plus de 60 degrés jour), alors qu'en eau douce il est en accord avec celui déjà observé par Escaffre et al. (1977) chez la truite arc-en-ciel.

En ce qui concerne les gamètes mâles, les spermes des animaux maintenus en mer induisent, en moyenne, une baisse importante du taux d'embryonnement quelle que soit l'origine des femelles. Cet élément, associé à la chute modeste de qualité des ovules des femelles maintenues en mer, pourrait expliquer la qualité médiocre des embryonnements observés à l'échelle de la production. C'est un fait intéressant car dans les études antérieures la mauvaise qualité des fécondations obtenues avec des géniteurs de Salmonidés, maintenus en mer, était le plus souvent attribuée aux ovules. Ce phénomène n'est probablement pas lié à un vieillissement précoce des spermatozoïdes car il est observé dès les premières fécondations. Les spermatocrites mesurés en 1986 laissent penser que la concentration en spermatozoïdes ne constitue pas un facteur limitant (Le Gac et al., 1986). Chez la truite arc-en-ciel, l'élévation des concentrations en ions $\mathrm{Na}^{+}$et du rapport $\mathrm{Na}^{+} / \mathrm{K}^{+}$lors de la spermiation a déjà été associée à l'effet du vieillissement (SanchezRodriguez et al., 1978). Lors de notre expérimentation, le maintien en mer des reproducteurs pendant la phase finale de la maturation sexuelle s'est aussi traduit par une osmolarité élevée du fluide séminal, une augmentation des concentrations en ions $\mathrm{Cl}^{-}$et une tendance à une élévation des ions $\mathrm{Na}^{+}$et $\mathrm{K}^{+}$et du rapport $\mathrm{Na}^{+} / \mathrm{K}^{+}$et par des concentrations prolactine et de $17 \alpha 20 \beta-\mathrm{OH}$-progestérone significativement plus basses que chez les mâles ramenés en eau douce (Le Gac et al., 1986). L'altération de qualité a pu être directement ou indirectement provoquée par ces modifications des statuts endocrinien et osmotique des animaux (Billard, 1983; Maisse et al., 1988). Il faut cependant noter que dans notre étude aucune corrélation entre les pressions osmotiques des fluides séminaux et les taux d'embryonnement individuels n'ont pu être mis en évidence.

Les résultats des fécondations effectuées à différentes pressions osmotiques montrent que si tous les spermes sont affectés par les osmolarités extrêmes ( $<200 \mathrm{mOsmol} / 1$ et $>350 \mathrm{mOsmol} / /$ ), les mauvais résultats obtenus avec certains spermes EM ne sont pas imputables à un éventuel choc osmotique lors 
de la mise en contact avec le dilueur de fécondation utilisé en routine (DIA532 $2^{(\bar{B}}$ à $250 \mathrm{mOsmol} / \mathrm{l}$ ). La modification de l'osmolarité du dilueur ne constitue donc pas une solution applicable en élevage. Enfin, l'absence de relations significatives entre la fécondance de spermes et l'osmolarité des fluides séminaux correspondants ne permet pas de retenir ce dernier critère comme paramètre d'évaluation $a$ priori de la qualité individuelle des spermes. Cependant, en l'absence de données sur la motilité des spermes, il ne peut être exclu une mise en mouvement prématurée des spermatozoïdes par dilution accidentelle des éjaculats avec de l'urine mais différentielle entre les deux milieux d'élevage des reproducteurs.

\section{Application}

En terme de gestion des géniteurs, il ressort de nos expériences que :

- L'utilisation des gamètes des reproducteurs mâles et femelles maintenus en mer jusqu'à la reproduction aboutit à des taux d'embryonnement moyens médiocres;

- Le transfert des géniteurs en eau douce 1 à 2 mois avant le début de la période de maturation des femelles permet d'éviter le retard et l'étalement des pontes observés en eau de mer et de produire des ovules et des spermes en quantité et en qualité très satisfaisante;

- Si les géniteurs ne peuvent être transférés en eau douce, il est suggéré d'éviter le vieillissement des ovules dans la cavité abdominale des femelles en surveillant les ovulations à intervalle de tris rapprochés inférieur à 60 degrés jours, l'utilisation d'inducteurs de la ponte pouvant réduire le travail nécessaire. Une deuxième proposition peut être introduite en incubant individuellement les pontes et en éliminant au stade oillé celles présentant de mauvais taux d'embryonnement du fait de la fécondation avec des lots de spermes ou des mâles de qualité médiocre. Ces améliorations pourraient être utilement complétées par une observation de la motilité initiale de chaque sperme et l'élimination des spermes non motiles, technique non introduite dans cette étude.

\section{Remerciements}

Nous exprimons tous nos remerciements aux techniciens de la SEMII et de la Station aquacole de St-Anne du Porzic du Centre IFREMER de Brest responsables de l'élevage des poissons.

\section{RÉFÉRENCES}

Billard R. 1977. A new technique of artificial insemination for Salmonids using a sperm diluent. Fisheries 1, 24-25.

Escaffre A. M., J. Petit, R. Billard 1977. Évolution de la quantité d'ovules et de la durée de la conservation de la fécondabilité durant la période post-ovulatoire chez la truite arc-en-ciel. Bull. Fr. Piscic. 265, 134-142.

Fontaine M. 1975. Physiological mechanisms in the migration of marine and amphihaline fish. Adv. Mar. Biol. 13, 241-355.

Hirano T., M. Morisawa, K. Susuki 1978. Changes in plasma and coelomic fluid composition of mature salmon Oncorhynchus keta during freshwater adaptation. Comp. Biochem. Physiol. 61, 5-8.

Harache Y. 1975. La salmoniculture marine en Norvège, état de développement en 1975. Rapp. scient. techn. CNEXO $\mathrm{n}^{\circ} 28$.

Harache Y., A. Faure 1986. L'élevage de la truite arc-en-ciel en mer. Piscic. Fr. 86, 38-43.

Le Bail P. Y. 1986. Growth-Reproduction interaction in Salmonids. In : Reproduction in fish-Basic and applied aspect in endocrinology and genetics. Les colloques de l'INRA 44, 91-108.
Le Gac F. 1986. Influence des conditions d'osmorégulation sur la reproduction du saumon Atlantique. Rapp. contrat INRA-IFREMER.

Maisse G., J. P. Porcher 1981. Une méthode de production de la truite fario pour le repeuplement. Bull. Fr. Piscic. 280, 134-144.

Maisse G., A. Pinson, M. Loir 1988. Caractérisation de l'aptitude à la congélation du sperme de truite arc-en-ciel par des critères physico-chimiques. Aquat. Living Resour. 1, 45-51.

Morisawa M., T. Hirano, K. Susuki 1979. Changes in blood and seminal plasma composition of mature salmon Oncorhynchus keta during adaptation to freshwater. Comp. Biochem. Physiol. 64, 325-329.

O'Flynn F. M., S. C. Backman, G. H. Friars 1992. The use of ovaprim at spawning. SGRP. Annual report of Atlantic Salmon Federation 1991/1992.

Prouzet P., J. L. Gaignon 1982. Fécondité des saumons atlantiques adultes capturés sur le bassin versant de l'Elorn (rivière de Bretagne Nord) et caractéristiques de leurs pontes. Bull. Fr. Piscic. 285, 233-243.

Quillet E., A. Faure, B. Chevassus, F. Krieg, Y. Harache, J. Arzel, R. Métailler, G. Bøuf 1992. The potential of brown trout Salmo trutta L. for mariculture in temperate waters. Buvisindi. Icel. Agr. Sci. 6, 63-77. 
Sanchez-Rodriguez M., A. M. Escaffre, S. Marlot, P. Reinaud 1978. The spermiation period in the rainbow trout (Salmo gairdneri). Plasma gonadotropin and androgen levels, sperm production and biochemical changes in the seminal fluid. Ann. Biol. Anim. Biochem. Biophys. 10, 943-948.

Sower S. A., C. B. Schreck $1982 a$. Steroid and thyroid hormones during sexual maturation of coho salmon Oncorhynchus kisutch in seawater or fresh water. Gen. Comp. Endocrinol. 67, 42-53.

Sower S. A., C. B. Schreck, E. M. Donaldson $1932 \mathrm{~b}$. Hormones-induced ovulation of coho salmon
Oncorhynchus kisutch held in seawater and fresh water. Can. J. Fish. Aquat. Sci. 3), 627-632.

Thorpe J. E. 1986. Age at first maturity in Atlantic salmon, Salmo salar: freshwater period infiuences and conflicts with smolting. Can. Spec. publ. Fish. Aquat. Sci. 7-14.

Wertheimer A. C. 1934. Maturation success of pink salmon Oncorhynchus gorbusha and coho salmon $O$. kisutch held under three salinity regimes. Aquaculture 43, 195-212.

Zohar Y. 1993. Manipulation of spawning in farmed fish. World Aquaculture'93, Torremolinos. European Aquaculture Society Spec. publ. 19, 509. 